Journal of Oral Science, Vol. 54, No. 1, 77-83, 2012

Original

\title{
Prevalence of Porphyromonas gingivalis fim $A$ genotypes in Japanese children
}

\author{
Fumiko Hayashi'), Mitsugi Okada2), Yuki Oda ${ }^{2}$, Taro Kojima²) and Katsuyuki Kozai ${ }^{1)}$ \\ 1)Department of Pediatric Dentistry, Hiroshima University Graduate School of Biomedical Sciences, \\ Hiroshima, Japan \\ 2)Department of Special Care Dentistry, Hiroshima University Hospital, Hiroshima, Japan
}

(Received 21 September 2011 and accepted 14 February 2012)

\begin{abstract}
Porphyromonas gingivalis FimA fimbriae have been classified into 6 genotypes (types I-V and Ib) based on the diversity of the fim $A$ genes encoding the fimbrial subunits. We investigated the prevalence of fim $A$ genotype in Japanese children. Dental plaque specimens were obtained from 400 subjects (age; 2 to 15 years), including 134 with healthy gingiva, 239 with gingivitis and 27 with periodontitis, and then analyzed by polymerase chain reaction. $P$. gingivalis was detected in $1.5 \%, 10.0 \%$ and $29.6 \%$ of these subjects, respectively. Significant differences were observed with regard to $P$. gingivalis infection among the groups [chi-squared analysis: gingivitis vs. healthy, $P<0.01$, odds ratio $(\mathrm{OR})=$ 7.4; periodontitis vs. healthy, $P<0.001, \mathrm{OR}=27.8$ ] In $P$. gingivalis-positive subjects with periodontitis, the most prevalent fim $A$ types were type Ib/type II combination $(37.5 \%)$ and type IV $(37.5 \%)$, followed by type II (25.0\%), while type IV (33.3\%) and type II $(29.2 \%)$ were most often detected in those with gingivitis. Our results suggest that the presence of $P$. gingivalis is associated with periodontal diseases, and that the type II, IV and Ib/II combination are the most common among fim $A$ genotypes. (J Oral Sci 54, 77-83, 2012)
\end{abstract}

Correspondence to Dr. Mitsugi Okada, Department of Special Care Dentistry, Hiroshima University Hospital, 1-2-3, Kasumi, Minami-ku, Hiroshima 734-8553, Japan

Tel: +81-82-257-5788

Fax: +81-82-257-5789

E-mail: mitsugi@hiroshima-u.ac.jp
Keywords: Porphyromonas gingivalis; children; fimA; genotype; periodontal condition.

\section{Introduction}

Periodontal diseases are mainly associated with gramnegative bacteria that initiate a series of events leading to the loss of periodontal attachment and alveolar bone surrounding teeth (1). Among these pathogens, Porphyromonas gingivalis is considered to be one of the most important oral cavity infectious agents, as it can cause several types of periodontal diseases (2). P. gingivalis is uncommon or found in low numbers in healthy individuals and those with gingivitis, while it is more frequently detected in those with more destructive forms of disease (3). The pathogen is considered to be mainly associated with adult periodontitis (4), and we previously found that $P$. gingivalis organisms were rarely present in the oral cavities of healthy children (5).

$P$. gingivalis has been shown to produce a number of virulence factors, including fimbriae, lipopolysaccharide, capsules and proteases (6). In particular, FimA fimbriae, filamentous components on the cell surface, are thought to play important roles in the colonization and invasion of periodontal tissue $(7,8)$. The $P$. gingivalis fimA gene has been classified into six variants (types I through $\mathrm{V}$ and $\mathrm{Ib}$ ) based their nucleotide sequences (9-11). A polymerase chain reaction (PCR) method with fimA typespecific primer sets has been developed to differentiate the six types of fimA genes of $P$. gingivalis in saliva and dental plaque samples collected from periodontitis patients $(11,12)$. Furthermore, the infectious traits of this bacterium that have been reported to have an influence 
Table 1 fimA type-specific and 16S ribosomal RNA-specific primers used in this study

\begin{tabular}{|c|c|c|c|c|}
\hline Primer pairs & Direction & Sequence & Size $(b p)^{a}$ & Reference \\
\hline \multirow{2}{*}{$\begin{array}{l}\text { P. gingivalis } \\
16 \mathrm{~S} \text { ribosomal RNA }\end{array}$} & forward & AGG CAG CTT GCC ATA CTG CG & \multirow{2}{*}{404} & \multirow{2}{*}{19} \\
\hline & reverse & ACT GTT AGC AAC TAC CGA TGT & & \\
\hline \multirow[t]{2}{*}{ Type I fimA } & forward & CTG TGT GTT TAT GGC AAA CTT C & \multirow{2}{*}{392} & \multirow{2}{*}{12} \\
\hline & reverse & AAC CCC GCT CCC TGT ATT CCG A & & \\
\hline \multirow[t]{2}{*}{ Type Ib fimA } & forward & CAG CAG AGC CAA AAA CAA TCG & \multirow{2}{*}{271} & \multirow{2}{*}{11} \\
\hline & reverse & TGT CAG ATA ATT AGC GTC TGC & & \\
\hline \multirow[t]{2}{*}{ Type II fimA } & forward & ACA ACT ATA CTT ATG ACA ATG G & \multirow{2}{*}{257} & \multirow{2}{*}{12} \\
\hline & reverse & AAC CCC GCT CCC TGT ATT CCG A & & \\
\hline \multirow[t]{2}{*}{ Type III fim $A$} & forward & ATT ACA CCT ACA CAG GTG AGG C & \multirow{2}{*}{247} & \multirow{2}{*}{12} \\
\hline & reverse & AAC CCC GCT CCC TGT ATT CCG A & & \\
\hline \multirow[t]{2}{*}{ Type IV fimA } & forward & CTA TTC AGG TGC TAT TAC CCA A & \multirow{2}{*}{251} & \multirow{2}{*}{12} \\
\hline & reverse & AAC CCC GCT CCC TGT ATT CCG A & & \\
\hline \multirow[t]{2}{*}{ Type V fimA } & forward & AAC AAC AGT CTC CTT GAC AGT G & \multirow{2}{*}{462} & \multirow{2}{*}{10} \\
\hline & reverse & TAT TGG GGG TCG AAC GTT ACT GTC & & \\
\hline \multirow{2}{*}{$\begin{array}{l}\text { Eubacterial } \\
16 \mathrm{~S} \text { ribosomal RNA }\end{array}$} & forward & CAG GAT TAG ATA CCC TGG TAG TCC ACG C & \multirow{2}{*}{625} & \multirow{2}{*}{12} \\
\hline & reverse & GAC GGG CGG TGT GTA CAA GGC CCG GGA ACG & & \\
\hline
\end{tabular}

a: Expected size of PCR product

on periodontal health status can be differentiated based on clonal variations of the fimA genes (13).

The prevalence of fimA genotypes in adults is reportedly related to periodontal condition. However, few investigations have noted a relationship between the prevalence of fimA genotypes and periodontal health status in children (14). The purpose of the present study was to investigate the prevalence of fimA genotype in Japanese children.

\section{Subjects}

\section{Materials and Methods}

Four hundred children, aged 2 to 15 years, who were patients at the Pediatric Dental Clinic of Hiroshima University Hospital, Japan, were enrolled in the present study. Consent for participation was obtained from at least one of their parents prior to the study according to the ethical guidelines of the Declaration of Helsinki (1975) and ethical clearance was obtained from the Ethical Committee of Hiroshima University (Epidemiology-No. $52 / 2$ ). Subjects with clinically healthy gingiva, as well as those with gingivitis and periodontitis, were studied and classified as follows (5). Those with an absence of inflammation, no bleeding on brushing, and no radiographic bone loss were regarded as having clinically healthy gingiva and classified as the healthy group ( $n=$ 134), while subjects with mild to moderate inflammation at more than 1 site, as determined by gingival index (15), and no radiographic bone loss were classified as the gingivitis group $(n=239)$, and those with attachment loss of greater than $3 \mathrm{~mm}$ at 1 site or more among four teeth were defined as the periodontitis group $(n=27)$. Children with systematic diseases were not excluded, although subjects who had taken antibiotics within 3 months prior to the study were excluded.

\section{Plaque sampling and genomic DNA preparation}

Dental plaque was collected from all erupted teeth by brushing with a sterile toothbrush for 1 minute, in accordance with a previously described method (5). Plaque samples were immediately transported to the laboratory and stored at $-20^{\circ} \mathrm{C}$ before extraction of genomic DNA. Genomic DNA from each plaque sample was obtained using a standard miniprep procedure, as reported previously (16), to which we added RNase treatment (17).

\section{PCR}

Table 1 shows the PCR primers used in the present study. A PCR method was used to detect $P$. gingivalis (16S ribosomal RNA), and fimA typing was then performed using species-specific primers, as described previously $(10-12,18)$. All primers were purchased from Sigma-Aldrich Japan (Tokyo, Japan). We also detected eubacterial 16S ribosomal RNA (GenBank accession number M75035) using a method described previously by Goncharoff et al. (19). Eubacterial $16 \mathrm{~S}$ ribosomal 
Table 2 Distribution of $P$. gingivalis in children divided into healthy, gingivitis, and periodontitis groups, and probability of $P$. gingivalis detection

\begin{tabular}{lcccccc}
\hline & \multicolumn{3}{c}{ P. gingivalis } & & & \multicolumn{2}{c}{ 95\% C. I. for odds ratio } \\
& Undetected & Detected (\%) & Chi-square & Odds ratio & Lower & Upper \\
\hline Healthy & 132 & $2(1.5)$ & & & & \\
Gingivitis & 215 & $24(10.0)$ & $9.68 * *$ & 7.4 & 1.7 & 31.7 \\
Periodontitis $^{*}$ & 19 & $8(29.6)$ & $30.54^{* * *}$ & 27.8 & 5.5 & 140.8 \\
\hline
\end{tabular}

‡vs. Healthy group, $* *: P<0.01, * * *: P<0.001$

Table 3 Distribution of $P$. gingivalis in children with healthy, gingivitis, and periodontitis in different dentition stages

\begin{tabular}{|c|c|c|c|c|}
\hline & & \multicolumn{3}{|c|}{ Number positive subjects/sampled subjects (\%) } \\
\hline \multicolumn{2}{|c|}{ Dentition (number of subjects) } & Healthy & Gingivitis & Periodontitis \\
\hline Primary & $(125)$ & $0 / 74 \quad(0.0)$ & $2 / 44 \quad(4.5)$ & $1 / 7 \quad(14.3)$ \\
\hline Mixed & $(215)$ & $2 / 47 \quad(4.3)$ & $17 / 152(11.8)$ & $4 / 16(25.0)$ \\
\hline Permanent & $(60)$ & $0 / 13 \quad(0.0)$ & $5 / 43 \quad(11.6)$ & $3 / 4 \quad(75.0)$ \\
\hline Total & $(400)$ & 2/134 (1.5) & $24 / 239(10.0)$ & $8 / 27(29.6)$ \\
\hline
\end{tabular}

RNA primers that match nearly all bacterial $16 \mathrm{~S}$ rRNA genes were used (18). PCR amplification was performed in a reaction mixture $(25 \mu \mathrm{L})$ consisting of PCR beads (GE Healthcare UK Limited, Buckinghamshire, UK) containing enzyme (two units of Taq DNA polymerase) and the required reagents, as well as 25 pmol of each primer, and 20 to $50 \mathrm{ng}$ of template DNA solution in a thermal cycler (DNA Engine PTC-220 DYAD; MJ Research, Waltham, MA, USA). Each set of PCR analyses included a negative control (water blank) in addition to the positive control. Reaction mixtures were denatured at $95^{\circ} \mathrm{C}$ for $5 \mathrm{~min}$, followed by 35 cycles of denaturation at $94^{\circ} \mathrm{C}$ for $30 \mathrm{~s}$, annealing at $58^{\circ} \mathrm{C}$ for $30 \mathrm{~s}$, and extension at $72^{\circ} \mathrm{C}$ for $30 \mathrm{~s}$, with a final cycle of $72^{\circ} \mathrm{C}$ for $7 \mathrm{~min}$ (13). After amplification, PCR products for fim $A$ type Ib were digested with RsaI (11). Next, $15 \mu \mathrm{L}$ of each PCR product was analyzed by electrophoresis on a 1.2-2.0\% agarose gel and the newly synthesized DNA fragments were visualized under a $302-\mathrm{nm}$ ultraviolet light, after staining with ethidium bromide. Digested PCR products for fimA type Ib were fragments of 162 and $109 \mathrm{bp}$. PCR detection limits were in the range of $25-100$ cells $(12,18)$. In the present study, the sensitivity of the PCR assay using the fimA type primer set was approximately 25-100 cells in plaque samples from the subjects, and PCR analysis using $16 \mathrm{~S}$ rDNA primer confirmed the presence of bacteria (data not shown).

\section{Statistical analysis}

Chi-squared test was used for statistical analysis of the comparative frequencies of bacterial occurrence among all periodontal groups. Statistical analyses were conducted using SPSS 10.0J (SPSS Inc., Chicago, IL, USA).

\section{Results}

Values showing the distribution of $P$. gingivalis in children in the healthy gingiva, gingivitis and periodontitis groups, as well as the ratios for probability of $P$. gingivalis detection, are shown in Table 2. Overall, $P$. gingivalis was detected in $2(1.5 \%)$ of 134 subjects in the healthy group, $24(10.0 \%)$ of 239 in the gingivitis group, and $8(29.6 \%)$ of 27 in the periodontitis group. Significant differences were observed in $P$. gingivalis infection among the groups [gingivitis group vs. healthy group, $P<0.01$, odds ratio $(\mathrm{OR})=7.4$; periodontitis group vs. healthy group, $P<0.001$, OR $=27.8]$.

The distributions of $P$. gingivalis in the healthy, gingivitis and periodontitis groups in different dentition stages are shown in Table 3. In the gingivitis group, P. gingivalis was detected in $17(11.8 \%)$ of 152 subjects with mixed dentition and $5(11.6 \%)$ of 43 with permanent dentition, while it was found in $2(4.5 \%)$ of 44 with primary dentition. In the periodontitis group, $P$. gingivalis was detected in $4(25.0 \%)$ of 16 subjects with mixed dentition and $3(75.0 \%)$ of 4 with permanent dentition, while it was found in $1(14.3 \%)$ of 7 with primary dentition. In contrast, $P$. gingivalis was found in only $2(4.3 \%)$ of 47 subjects in the healthy group with mixed dentition, while the organism was not isolated from any of the healthy subjects with either primary or permanent dentition.

The distribution of $f i m A$ genotypes among $P$. gingivalis organisms found in the plaque samples was subsequently examined. Table 4 shows the distribution of the 6 fim $\mathrm{A}$ 
Table 4 Distribution of 6 fimA types in children possessing $P$. gingivalis divided into healthy, gingivitis, and periodontitis groups

\begin{tabular}{lcccc}
\hline & \multicolumn{4}{c}{$\begin{array}{c}\text { Percent frequency of occurrence } \\
\text { (No. of positive subjects) }\end{array}$} \\
\cline { 2 - 5 } fim A type & Healthy $(2)$ & Gingivitis $(24)$ & Periodontitis $(8)$ & Total $(34)$ \\
\hline I & 0 & 0 & 0 & 0 \\
Ib & 0 & 0 & 0 & 0 \\
II & $50.0(1)$ & $29.2(7)$ & $25.0(2)$ & $29.4(10)$ \\
III & 0 & $8.3(2)$ & 0 & $5.9(2)$ \\
IV & 0 & $33.3(8)$ & $37.5(3)$ & $32.4(11)$ \\
V & 0 & 0 & 0 & 0 \\
Ib+II & $50.0(1)$ & $16.7(4)$ & $37.5(3)$ & $23.5(8)$ \\
Ib+II+V & 0 & $4.2(1)$ & 0 & $2.9(1)$ \\
Untypeable & 0 & $8.3(2)$ & 0 & $5.9(2)$ \\
\hline
\end{tabular}

Table 5 Distribution of 6 fimA types in children possessing $P$. gingivalis divided into different dentition stages

\begin{tabular}{lcccc}
\hline & \multicolumn{4}{c}{$\begin{array}{c}\text { Percent frequency of occurrence } \\
\text { (No. of positive subjects) }\end{array}$} \\
\cline { 2 - 4 } fimA type & \multicolumn{3}{c}{ Dentition } & Total (34) \\
\cline { 2 - 4 } I & Primary (3) & Mixed (23) & Permanent (8) & 0 \\
Ib & 0 & 0 & 0 & 0 \\
II & 0 & 0 & 0 & $29.4(10)$ \\
III & $66.7(2)$ & $21.7(5)$ & $37.5(3)$ & $5.9(2)$ \\
IV & 0 & $8.7(2)$ & 0 & $32.4(11)$ \\
V & $33.3(1)$ & $30.4(7)$ & $37.5(3)$ & 0 \\
Ib+II & 0 & 0 & 0 & $23.5(8)$ \\
Ib+II+V & 0 & $26.1(6)$ & $25.0(2)$ & $2.9(1)$ \\
Untypeable & 0 & $4.4(1)$ & 0 & $5.9(2)$ \\
\hline
\end{tabular}

types in subjects in the healthy, gingivitis and periodontitis groups who possessed $P$. gingivalis. Overall, a single fim $A$ genotype was detected in $67.7 \%$ of all subjects, with type IV most frequently detected $(32.4 \%)$, followed by type II (29.4\%). Multiple fimA genotypes were detected in $26.4 \%$ of all subjects. Among all $P$. gingivalis-positive periodontitis subjects, the most prevalent fimA types were type Ib/type II combination $(37.5 \%)$ and type IV (37.5\%), followed by type II $(25.0 \%)$. Subjects with gingivitis predominantly possessed type IV (33.3\%) and type II (29.2\%). In contrast, type I was not detected in any of the subjects, while the type Ib was also not detected as a single fim $A$ genotype. Untypeable strains were detected in $5.9 \%$ of all subjects, while type Ib was not detected in any subjects with primary dentition. Table 5 shows the distribution of the 6 fim $A$ types in children possessing $P$. gingivalis divided into different dentition stages. Type II and type IV were detected in subjects with all types of dentition, while type Ib/II combination was only detected in subjects with mixed and permanent dentition.

\section{Discussion}

We investigated the prevalence of $P$. gingivalis in 400 children divided into healthy, gingivitis and periodontitis groups, with the pathogen detected in $1.5 \%, 10.0 \%$ and $29.6 \%$ of these subjects, respectively. Mättö et al. (20) reported that the detection frequency of $P$. gingivalis was $5 \%$ in saliva samples from subjects aged 5 to 10 years and $14 \%$ in those from subjects aged 11 to 20 . We previously found that $P$. gingivalis was rarely present in the oral cavities of healthy children aged 2 to 12 years (5), of whom $4.8 \%$ of the subjects $(0 \%$ with primary dentition and $11.1 \%$ with mixed dentition) were positive for $P$. gingivalis in plaque samples obtained by toothbrushing. Tamura et al. (14) also reported that samples from 3.2\% of children and adolescents without periodontitis showed a positive reaction to $P$. gingivalis-specific primers, while Ashimoto et al. (18) found that the prevalence of 
P. gingivalis in children aged 2 to 11 years with pediatric gingivitis was $14 \%$. In the present study, we used a PCR method and detected $P$. gingivalis in children aged 2 to 15 years, and our results agreed with those of previous reports $(5,14,18)$. Furthermore, the prevalence of $P$. gingivalis was significantly different among the present healthy, gingivitis and periodontitis groups, and we found evidence that the pathogen may be directly related to periodontitis in children. When we divided the periodontitis group based on stage of dentition, those with permanent dentition had the highest rate $(75.0 \%)$ of occurrence, as compared to those with mixed $(25.0 \%)$ and primary $(14.3 \%)$ dentition. Our findings also agree with previous studies that reported a significant correlation between age and rate of detection of $P$. gingivalis $(14,21)$.

In the present study, type Ib/type II fimA combination $(37.5 \%)$ and type IV (37.5\%) were predominant in children with periodontitis, followed by type II (25.0\%). Nakagawa et al. (11) reported that type Ib/type II fimA combination was not present in any adults tested. Thus, the prevalence of this $P$. gingivalis fimA combination in children may be different from that in adults based on periodontal condition. Tamura et al. (14) later noted that none of their subjects were type II fim $A$ positive. However, we found that 10 of 34 (29.4\%) of our subjects who possessed $P$. gingivalis were type II fimA-positive. According to Missailidis et al. (22) and Miura et al. (23), the most prevalent fim $A$ type in adult periodontitis patients is type II, followed by type Ib. In addition, Amano (8) reported that the majority of adult periodontitis patients harbor type II and that the next most prevalent is type IV. These results for adult periodontitis patients were similar to the present results obtained in children. We also reported previously that the prevalence of $P$. gingivalis was $71.4 \%$ in both fathers and mothers of proband families, while it was $35.7 \%$ in their children (24), and we found that parents are an important source of periodontal pathogens that colonize the oral cavities of their children (24). On the other hand, $P$. gingivalis was shown to be less easily transmitted from parents to children (25), while another study noted that the pathogen may be difficult to transmit or require a longer period of time for colonization (26). Asano et al. (27) also suggested that fimA type II, widely distributed in patients with periodontitis, may be an important factor in the transmission of $P$. gingivalis between spouses.

In the present $P$. gingivalis-positive gingivitis subjects, the most prevalent fimA type was type IV (33.3\%), followed by type II (29.2\%). In a study conducted in Brazil, Missailidis et al. (22) reported that those with gingivitis commonly had type I/type IV fimA combination, followed by subjects with type IV. It is possible that type IV and type II are related to gingivitis, as well as periodontitis, in children.

Several studies have shown that the most prevalent fimA genotype in healthy adults is type I $(8,12,13,23)$, while organisms with types II and IV were found to be prevalent in only $9.4 \%$ and $6.5 \%$, respectively, of studied subjects (13). However, in the present study, type I was not detected in any of the subjects, and type $\mathrm{Ib}$ and type $\mathrm{V}$ were not detected as a single fimA genotype. Nevertheless, longitudinal studies are required to evaluate the risk of occurrence and development of periodontitis in children.

In our study, untypeable fim $A$ was found in 2 children $(8.3 \%)$ with gingivitis, but not in any subjects with healthy gingiva or periodontitis. Tamura et al. (14) noted that one of the major characteristics of fim $A$ genotype distribution in children and adolescents is a high frequency of untypeable strains $(33.3 \%)$. On the other hand, Nakagawa et al. (11) reported that untypeable strains were found in adult subjects with $(1.5 \%)$ and without $(4.3 \%)$ periodontitis. Furthermore, Asano et al. (27) reported that untypeable strains were found in $6.3 \%$ of the adults with periodontitis. The discrepancies among these studies might be due, in part, to the sampling methods employed. However, further studies are required in order to better understand the virulence of untypeable fimA.

In summary, our findings indicate that the presence of $P$. gingivalis is associated with periodontal diseases, and that the type II, IV and $\mathrm{Ib} / \mathrm{II}$ combination are the most common among fimA genotypes.

\section{Acknowledgments}

We would like to thank Dr. Makoto Kawamura of Hiroshima University Hospital for his advice regarding statistical methods. This work was supported in part by a Grant-in-Aid for Scientific Research from the Ministry of Education, Science, Sports and Culture of Japan (16791287).

\section{References}

1. Zambon JJ (1994) Familial transmission of periodontal pathogens as a risk factor for periodontal disease progression. Compendium 15, 996-1000.

2. Darveau RP, Tanner A, Page RC (1997) The microbial challenge in periodontitis. Periodontol $200014,12-32$.

3. Haffajee AD, Socransky SS (1994) Microbial etiological agents of destructive periodontal diseases. Periodontol 2000 5, 78-111. 
4. Darby I, Curtis M (2001) Microbiology of periodontal disease in children and young adults. Periodontol 2000 26, 33-53.

5. Okada M, Hayashi F, Nagasaka N (2000) Detection of Actinobacillus actinomycetemcomitans and Porphyromonas gingivalis in dental plaque samples from children 2 to 12 years of age. J Clin Periodontol 27, 763-768.

6. Holt SC, Kesavalu L, Walker S, Genco CA (1999) Virulence factors of Porphyromonas gingivalis. Periodontol 2000 20, 168-238.

7. Takahashi Y, Yoshimura F, Kawanami M, Kato $\mathrm{H}$ (1992) Detection of fimbrilin gene (fimA) in Porphyromonas (Bacteroides) gingivalis by Southern blot analysis. J Periodontal Res 27, 599-603.

8. Amano A (2003) Molecular interaction of Porphyromonas gingivalis with host cells: implication for the microbial pathogenesis of periodontal disease. J Periodontol 74, 90-96.

9. Hamada S, Fujiwara T, Morishima S, Takahashi I, Nakagawa I, Kimura S, Ogawa T (1994) Molecular and immunological characterization of the fimbriae of Porphyromonas gingivalis. Microbiol Immunol 38, 921-930.

10. Nakagawa I, Amano A, Kimura RK, Nakamura T, Kawabata S, Hamada S (2000) Distribution and molecular characterization of Porphyromonas gingivalis carrying a new type of fimA gene. J Clin Microbiol 38, 1909-1914.

11. Nakagawa I, Amano A, Ohara-Nemoto Y, Endoh N, Morisaki I, Kimura S, Kawabata S, Hamada S (2002) Identification of a new variant of fimA gene of Porphyromonas gingivalis and its distribution in adults and disabled populations with periodontitis. J Periodontal Res 37, 425-432.

12. Amano A, Nakagawa I, Kataoka K, Morisaki I, Hamada S (1999) Distribution of Porphyromonas gingivalis strains with fimA genotypes in periodontitis patients. J Clin Microbiol 37, 1426-1430.

13. Amano A, Kuboniwa M, Nakagawa I, Akiyama S, Morisaki I, Hamada S (2000) Prevalence of specific genotypes of Porphyromonas gingivalis fimA and periodontal health status. J Dent Res 79, 1664-1668.

14. Tamura K, Nakano K, Nomura R, Miyake S, Nakagawa I, Amano A, Ooshima T (2005) Distribution of Porphyromonas gingivalis fimA genotypes in Japanese children and adolescents. J Periodontol 76, 674-679.

15. Löe H, Silness J (1963) Periodontal diseases in pregnancy I. Prevalence and severity. Acta Odontol Scand 21, 533-551.

16. Wilson K (1990) Preparation of genomic DNA from bacteria. In: Current protocols in molecular biology, Ausubel FM, Brent R, Kingston RE, Moore DD, Seidman JG, Smith JA, Struhl K eds, Greene Publishing Associated and WileyInterscience, New York, 2.4.1-2.4.2.

17. Smith GLF, Socransky SS, Smith CM (1989) Rapid method for the purification of DNA from subgingival microorganisms. Oral Microbiol Immunol 4, 47-51.

18. Ashimoto A, Chen C, Bakker I, Slots J (1996) Polymerase chain reaction detection of 8 putative periodontal pathogens in subgingival plaque of gingivitis and advanced periodontitis lesions. Oral Microbiol Immunol 11, 266-273.

19. Goncharoff P, Figurski DH, Stevens RH, Fine DH (1993) Identification of Actinobacillus actinomycetemcomitans: polymerase chain reaction amplification of 1ktA-specific sequences. Oral Microbiol Immunol 8, 105-110.

20. Mättö J, Saarela M, Alaluusua S, Oja V, JousimiesSomer H, Asikainen S (1998) Detection of Porphyromonas gingivalis from saliva by PCR by using a simple sample-processing method. J Clin Microbiol 36, 157-160.

21. Ooshima T, Nishiyama N, Hou B, Tamura K, Amano A, Kusumoto A, Kimura S (2003) Occurrence of periodontal bacteria in healthy children: a 2-year longitudinal study. Community Dent Oral Epidemiol 31, 417-425.

22. Missailidis CG, Umeda JE, Ota-Tsuzuki C, Anzai D, Mayer MPA (2004) Distribution of fimA genotypes of Porphyromonas gingivalis in subjects with various periodontal conditions. Oral Microbiol Immunol 19, 224-229.

23. Miura M, Hamachi T, Fujise O, Maeda K (2005) The prevalence and pathogenic differences of Porphyromonas gingivalis fimA genotypes in patients with aggressive periodontitis. J Periodontal Res 40, 147-152.

24. Okada M, Hayashi F, Soda Y, Zhong X, Miura K, Kozai K (2004) Intra-familial distribution of nine putative periodontopathogens in dental plaque samples analyzed by PCR. J Oral Sci 46, 149-156.

25. Asikainen S, Chen C, Slots J (1996) Likelihood of transmitting Actinobacillus actinomycetemcomitans and Porphyromonas gingivalis in families with periodontitis. Oral Microbiol Immunol 11, 387-394. 
26. Umeda M, Miwa Z, Takeuchi Y, Ishizuka M, Huang Y, Noguchi K, Tanaka M, Takagi Y, Ishikawa I (2004) The distribution of periodontopathic bacteria among Japanese children and their parents. J Periodontal Res 39, 398-404.
27. Asano $H$, Ishihara $K$, Nakagawa $T$, Yamada $S$, Okuda K (2003) Relationship between transmission of Porphyromonas gingivalis and fimA type in spouses. J Periodontol 74, 1355-1360. 